\title{
Testing Thermal Properties of the Cooling Device with Heat Pipes
}

\author{
P. Nemec ${ }^{1 \mathrm{a}}$, A. Čaja ${ }^{1}$, M. Malcho ${ }^{1}$ \\ ${ }^{1}$ University of Žilina, Department of Power Engineering, Univerzitná 1, 01026, Žilina, Slovakia
}

\begin{abstract}
Paper deal about testing of device with heat pipes and about research alternative possible applications of heat pipes and potential improvements in purpose effective heat sink from power switches of device. This device is used in various static and moveable applications. Testing device is part of control unit in rail vehicle. The amount of heat produced by power switches depend from working conditions of static converter. Great impact on heat sink has, how as season also this if the vehicle is moving or don't moving. If the vehicle is moving the heat transfer form energy converter to surrounding is caused by forced convection of air flow around device. But the critical conditions occur if the vehicle doesn't move, because the application of this cooling device is based on natural convection. The study deal solution of the problem heat transfer from energy converter to surrounding by natural convection.
\end{abstract}

\section{Introduction}

Capillary-driven two-phase systems offer significant advantages over traditional single phase systems and heat transfer systems by and thermal conductivity. With the typically increased thermal capacity associated with the phase change of a working fluid, considerably smaller mass flow rates are required to transport equivalent amounts than in other heat transfer systems for a given temperature range. Moreover, heat transfer coefficients of two-phase systems are much greater than others systems and result in enhanced heat transfer. Lower mass flow rates and enhanced thermal characteristics provide the benefits of smaller system size (and weight) while providing increased performance. The thermal capacity of a single-phase system depends on the temperature change of the working fluid; thus, a large temperature gradient or a high mass flow rate is required to transfer a large amount of heat. However, a two-phase system can provide essentially isothermal operation regardless of variations in the heat load. Additionally, single phase systems require the use of mechanical pumps and fans to circulate the working fluid, while capillary-driven twophase systems have no external power requirements, which make such systems more reliable and free of vibration. Heat pipes are passive devices that transport heat from a heat source (evaporator) to a heat sink (condenser) over relatively long distances via the latent heat of vaporization of a working fluid [1]. The concept of the heat pipe was first presented by [2,3], but was not widely publicized until an independent development by
[4] at the Los Alamos Scientific Laboratories. From the time of heat pipe patent and with this join using the latent heat of substance to heat transfer by phase change in hermetic closed vacuum system, is whereby further more applications in praxis, which use two phase systems to heat transfer. And whereby further more industrial branch try compose the heat pipe into its heat transfer process or operation of thermal devices in order to cooling or temperature stabilization. The most widespread branch of heat pipe applications is still electrical engineering, where the heat pipes are used to cooling power switches. This situation make researcher in the area of heat pipe research and development do test of various kinds and type configurations heat pipes for these applications. In this soul is written the article.

\section{Measurement of the cooling device}

At first was designed thermostatic chamber to testing of cooling device. The chamber is designed and constructed for simulation surrounding temperature conditions and must have a sufficient capacity for creating conditions for heat transfer by natural convection to simulate the adjustable and continuously stationary temperatures around cooling device. Construction and measuring in thermostatic chamber describe $[5,6]$.

patrik.nemec@fstroj.uniza.sk 

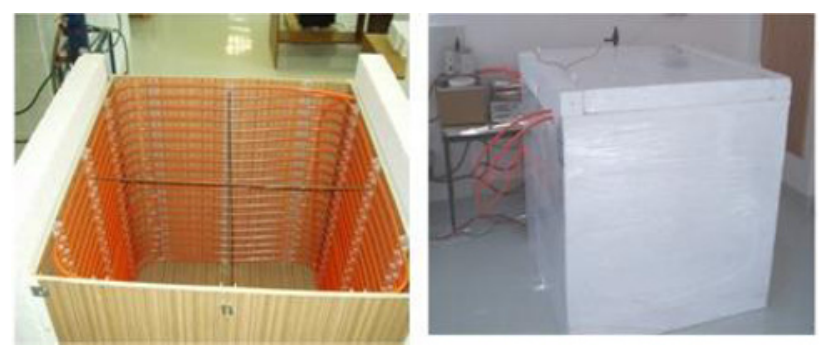

Fig. 1 Thermostatic chamber for testing cooling by natural convection.

The cooling device consists from energy converter and cooler. The cooler was constructed from six gravitational pipes filled with ethyl alcohol. Their evaporation section is embedded into the aluminium block of the energy converter and on the condensation section are ribs connected vertically to the axis of the heat pipes. The energy converter consist from electronic components, which are plug-in the electrical source and heat flow produced by electronic components is transported by heat pipes to the ribbing section. Head point testing of cooling device in thermostatic chamber is monitoring temperature on the aluminium block of energy converter, heat pipes and ribs at temperature condition $30{ }^{\circ} \mathrm{C}$ and various positions of device. On figure 2 is seen tilted energy converter about $20^{\circ}$ plug-in electric source and graphic results from the measurement. The input heat flux is 450 $\mathrm{W}$ and the temperature of the surrounding air in thermostatic chamber is $30^{\circ} \mathrm{C}$. Measurement of the device were at three different positions an tilt angle about $0^{\circ}, 10^{\circ}$ and $20^{\circ}$ from the vertical level. Intention of the measurement was to ascertain dependence of the heat transferred upon tilt angle of the cooling device with gravitational heat pipes from the vertical level. Therefore to determination efficiency of the cooling device from the working position, thermocouples scanned surface temperature course on the each heat pipes, the ribs of the ribbed cooler and the aluminum block of the energy converter. All the temperatures data were recorded into a computer by a measurement central. To determine electrical performance of electrical source and total performance of the cooling device was measured electric current and voltage of the heat source and input and output temperature and mass flow of the circulate fluid in thermostatic chamber.
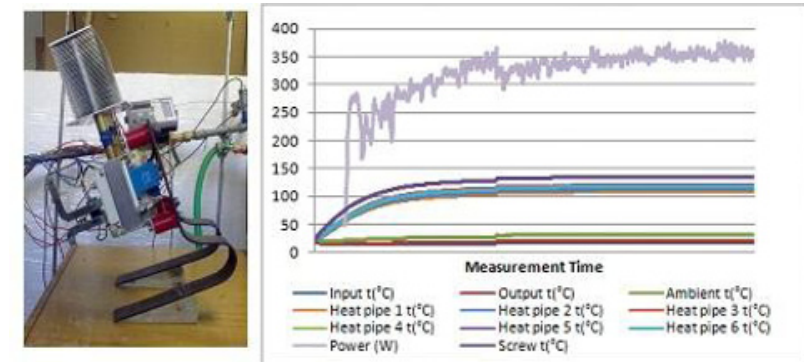

Fig.2 Graphic results from the measurement of the cooling device at tilt angle $20^{\circ}$ and outside temperature $30^{\circ} \mathrm{C}$

In all the three position was the highest temperature measured on the aluminum block of the device and on the figure 3 is shown their temperature course. At vertical position was the highest temperature measured $142{ }^{\circ} \mathrm{C}$, at second position at the inclination $10^{\circ}$ from vertical position was the highest measured temperature $136{ }^{\circ} \mathrm{C}$ and at third position at inclination $20^{\circ}$ from vertical position was the highest measured temperature $132{ }^{\circ} \mathrm{C}$. Temperature drop in the same point between vertical position of the cooling device and position at inclination $20^{\circ}$ from vertical position is approximately $10^{\circ} \mathrm{C}$.

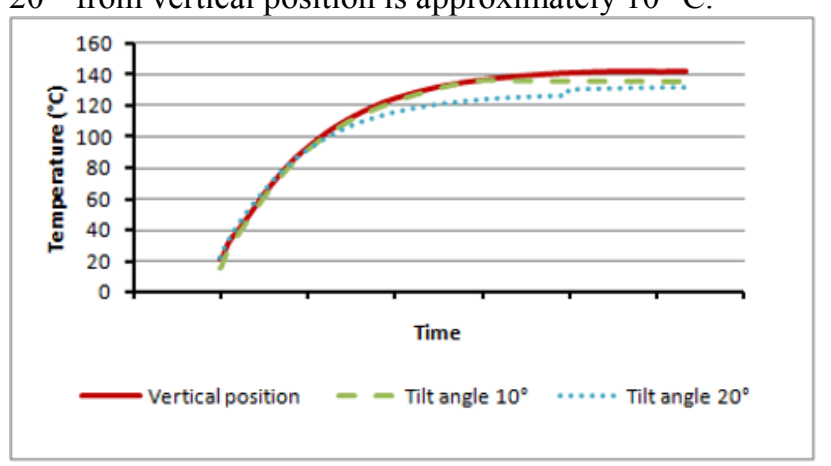

Fig.3 Temperature course at the cooling device at various positions

Result of the testing is that, the increasing of the tilt angle of the cooler device is possible to increase its ability to heat transfer but only up to a certain point. After exceed the critical point heat transfer ability of the cooler device start decrease. Therefore if the cooler is tilted the ribs are tilted in a certain angle from the vertical level too and in that way the heat transfer by natural convection increase too. Increase the tilt angle is possible only up to the point when the condensate in heat pipes is still able return from condensation section to the evaporation section. At a higher tilt angle position of the ribbed cooler is cooled more intensively by natural convection but the return of the condensate to the evaporator section fail and that is reason why its overall ability of the heat transfer decreases. Option how to improve heat transfer by natural convection to the surrounding is application of the wick heat pipe because the wick heat pipe is able operate at tilt angle $45^{\circ}$ position without any problems. This claim confirms study about influence working position of wick heat pipe on their performance.

\section{Measurement of wick heat pipes}

As an alternative application for heat transfer improvement of cooling device were manufactured and tested on our workplace department copper heat pipes with sintered capillary structure. For this experiment was made three various capillary structures by sintering from copper powders with granularity of $100 \mu \mathrm{m}, 63 \mu \mathrm{m}$ and $50 \mu \mathrm{m}$ applying the copper powder to the inner wall of the copper pipe. Capillary structure of heat pipes from cooper powder was sintered in the high thermal electric oven using for powder metallurgy. By this method was created a $1.5 \mathrm{~mm}$ thick coat of sintered capillary structure. The overall length of the heat pipes is $0,45 \mathrm{~m}$. 


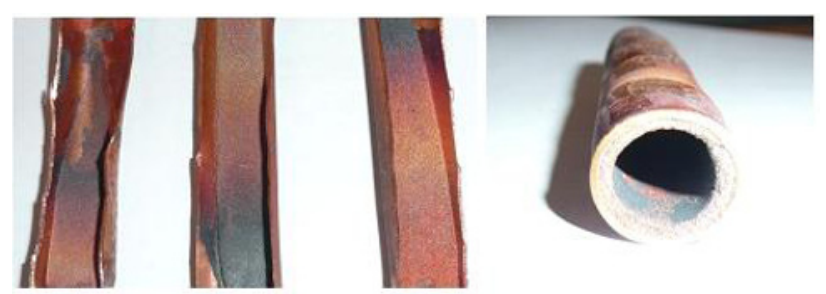

Fig.4 Wick heat pipe with sintered capillary structure

The choices of the capillary system for the wick heat pipes depend from many factors and only a few of these factors are related to the properties of the working fluid. The working fluid must have good thermal stability in relation to the specific working temperature and pressure. The most important requirements which the working fluid must have are the following: compatibility with the capillary system and with the material of the pipe, high thermal stability, high state of heat, high thermal conductivity, low viscosity of the liquid and vapour phase, high surface tension, acceptable freezing point. Therefore was as a working fluid chosen water and ethanol. Amount of working fluid in heat pipes is $20 \%$ from overall heat pipe volume as discuses [7, 8]. Working fluid was injected in to the pipe via connecting capillary by syringe. Pipe with working fluid was connect by vacuum sucking disk to vacuum system and by vacuum pump was inner air from pipe sucking off. Before connecting pipe to vacuum system had to cool working fluid by immersion of pipe in to the cooling medium, because during vacuuming of the pipe occur pressure drop and this may cause evaporate of working fluid. As a cooling medium may be used dry ice or liquid nitrogen. After vacuuming was connecting capillary cramping, cutting from vacuum system and end of heat pipe was soldering.

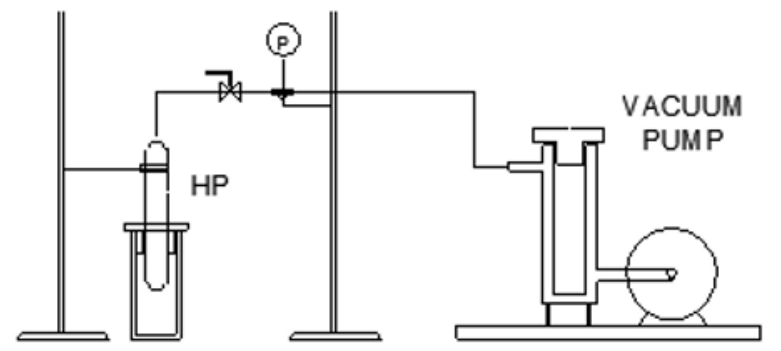

Fig.5 Schema of vacuuming rig

Heat pipe is simple but ingenious device to heat transfer. Heat transfer by heat pipe occur based on evaporation and consequential condensation of working fluid. After this meaner is possible to transfer great thermal performance by little dimensions devices, too. One from many methods how to determine performance of heat pipe is calorimetric method, which was used in experimental measurement by [9]. Calorimetric method emanating from calorimetric equation where known mass flow, specific heat capacity, input and output temperature of coolant. Total heat power of heat pipe determine from difference between input and output temperature of circumfluent coolant. For the experimental measurement was proposed measuring unit, which consist from measuring apparatus (thermostat, measuring centre, ultrasonic flowmeter, auto-transformer) necessary to measuring thermal performance of heat pipe.

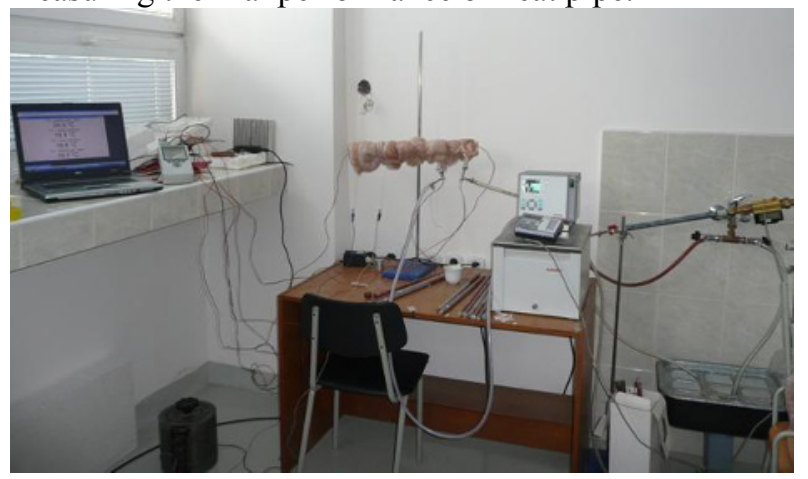

Fig. 6 Experimental measurement of heat pipe performance

Heat source for the heat pipe was controllable transformer, which was connected with resistance wire to heat pipe. Temperature of heat pipe evaporator section was measuring with $\mathrm{NiCr}-\mathrm{Ni}$ thermocouple taped on heat pipe surface. Electrical isolation under resistance wire constituted mica tape. Evaporator section of heat pipe was heating by resistance wire connecting on the transformer, which regulates necessary working temperature of heat pipe. To determine of heat pipe performance was designed cooling system consisted from small copper pipe placing on condenser section of heat pipe. To better heat transfer from heat pipe to cooling system was surface of het pipe painted with heatconductive paste. Through the cooling system flow cooling water and is regulated by thermostat. The input and output temperature flowing water through cooling system is measured by $\mathrm{NiCr}-\mathrm{Ni}$ thermocouples. Adiabatic and condensate section of heat pie is isolated by polystyrene as a heat loss protection into surround. The temperature and mass flow data from measuring enter into measuring centre and by software AMR to PC. Heat performance transferred from evaporator to condensate section by heat pipe is calculated based on calorimetrical equation from measured value of input and output temperature, mass flow and special thermal capacities cooling water circulate in cooling system [10]. Measurement of thermal performance of heat pipes was at vertical and tilt angel $45^{\circ}$ position and temperature of heat source $50{ }^{\circ} \mathrm{C}$ and $70{ }^{\circ} \mathrm{C}$. Heat performance solution of heat pipe is based on calorimetrical equation and values from experimental measuring. The same calculations were used at work [11].

$$
\begin{aligned}
& Q=\dot{m} c \Delta t \\
& \Delta t=t_{2}-t_{1}
\end{aligned}
$$

Where is $\Delta \mathrm{t}\left[{ }^{\circ} \mathrm{C}\right]$ - temperature difference, $\mathrm{t}_{1}\left[{ }^{\circ} \mathrm{C}\right]-$ input temperature, $\mathrm{t}_{2}\left[{ }^{\circ} \mathrm{C}\right]$ - output temperature, $\dot{\mathrm{m}}\left[\mathrm{J} \mathrm{kg}^{-1} \mathrm{~K}^{-1}\right]-$ mass flow of liquid, $\mathrm{c}\left[\mathrm{J} \mathrm{kg} \mathrm{s} \mathrm{s}^{-1}\right]-$ special thermal capacities of liquid.

On figure 7 are shown result from experimental measuring heat performance of wick heat pipe with sintered capillary structure from copper powder $63 \mu \mathrm{m}$ granulate at vertical position. 


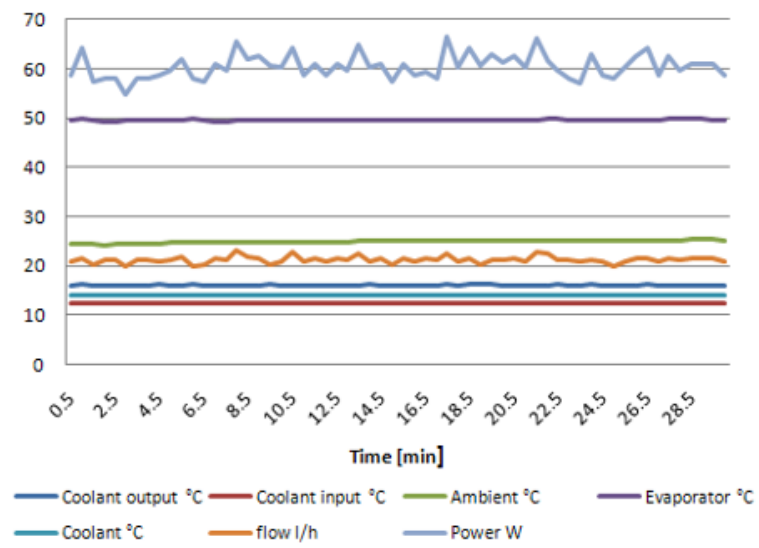

Fig.7 Results from experimental measuring heat performance of wick heat pipe with sintered capillary structure from copper powder $63 \mu \mathrm{m}$ granulate at vertical position

On the figure 8 is shown test result of wick heat pipes with sintered capillary structure from copper powders with granularity of $100 \mu \mathrm{m}, 63 \mu \mathrm{m}$ and $50 \mu \mathrm{m}$. In graph is declared percentage performance of this wick heat pipe at tilt angle $45^{\circ}$ from vertical position and it is seen, that the operate performance all various kinds of wick heat pipes were more than $80 \%$.

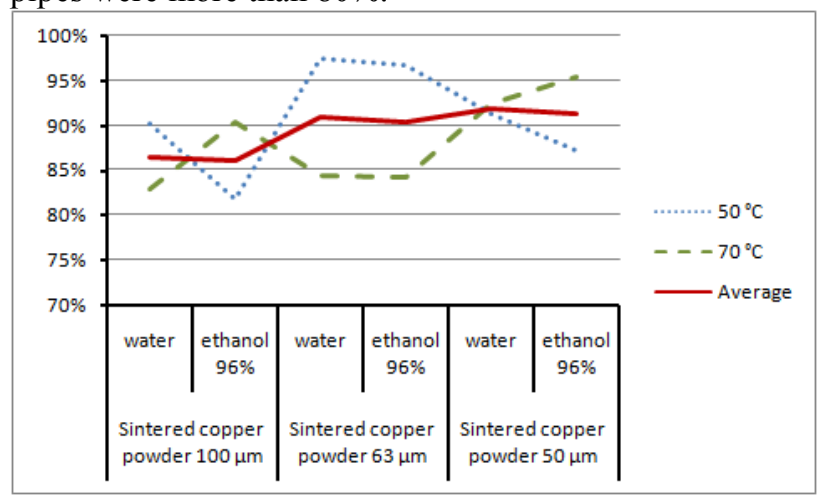

Fig.8 Percentage performance of sintered wick heat pipes at vertical and tilt angel $45^{\circ}$ position and temperature of heat source $50^{\circ} \mathrm{C}$ and $70{ }^{\circ} \mathrm{C}$

\section{Conclusion}

From experimental measuring thermal performance of wick heat pipes was create graphic dependence average values of thermal performance from working position and capillary structure. Ideal working position of heat pipe is vertical position. Heat pipe operate on maximum performance and maximum mass flow transfer in this position. This experiment has testify that the wick heat pipe is able operate at any other position for example at angle $45^{\circ}$ position. This phenomenon is caused by capillary action in capillary structure of heat pipe. Capillary effect caused return of substance condensate from condensation section to evaporation section of heat pipe. This process caused substance circulation in pipe needed to heat transfer by heat pipe. Substitution of wick heat pipes instead of gravitation heat pipes and tilt of cooling device will be occur better heat transfer by natural convection from ribbed cooler to the surrounding and heat transfer from source by wick heat pipes will be approximately the same.

\section{Acknowledgment}

This article was created within the frame of project APVV - $0448-07$ and 064ŽU-4/2012.

\section{References}

1. M. J. Ochterbeck, Heat Pipes in Heat Transfer Handbook, New York

2. R. S. Gaugler, Heat Transfer Devices, Dayton

3. L. Trefethen, On the surface tension pumping of liquids or a possible role of the candlewick in space exploration, New York

4. G. M. Grover, T. P. Cotter, G. F. Erikson, J. Appl. Phys., 35,1900, (1964)

5. A. Kapjor, J. Hužvár, P. Pilát, ERIN, 5, 57-60, (2011)

6. J. Hužvár, J. Jandačka, A. Oksanen, Optimization using exergy-based methods and computational fluid, 219-224, (2009)

7. R. Lenhard, Power control and optimization, 4, 13, (2010).

8. R. Lenhard, K. Kaduchová, S. Gavlas, J., Jandačka, HEAT, 6, 445-448, (2011)

9. A. Kovalčík, TRANSCOM, 16 - 19, (2009)

10. P. Pilát, J. Mičicová, TRANSCOM, 117-119, (2009)

11. A. Kovalčík, E. Toporcer, V. Hlavňa, Communications, 13, 43 - 46, (2011) 